\title{
ESTUDIO PROSPECTIVO SOBRE LA TASA DE DETECCIÓN DE CÁNCER DE PRÓSTATA EN NUESTRO HOSPITAL EN PACIENTES DEL NORESTE DE MÉXICO CON ANTÍGENO PROSTÁTICO ESPECÍFICO ENTRE 2.6 Y 4 NG/ML.
}

\author{
Jorge A. Arratia-Maqueo, Jeff R. Cortés-González, Roberto Garza-Cortes y Lauro S. \\ Gómez-Guerra.
}

Servicio de Urología. Hospital Universitario Dr. José E. González. UANL. Monterrey. Nuevo León. Méjico.

\begin{abstract}
Resumen.- OBJETIVO: Encontrar la tasa de detección de Cáncer Prostático (CaP) en nuestra población con Antígeno Prostático Específico (APE) entre 2.6 y 4 ng/ $\mathrm{ml}$.
\end{abstract}

MÉTODOS: Se incluyeron 33 pacientes consecutivos, mediana 66 años. se les realizó biopsia transrectal guiada por ultrasonido (BTR-US) con APE entre 2.6 y 4 $\mathrm{ng} / \mathrm{ml}$. Se dividieron en 2 grupos. Grupo 1: pacientes con tacto rectal (TR) normal. Grupo 2: pacientes con ligero aumento en la consistencia de la próstata. Criterios de exclusión: CaP, neoplasia intraepitelial o TR evidente de CaP. Análisis estadístico: $t$ de student, Fischer y X².

RESULTADOS: El 28.3\% (9) fue positiva para CaP. El $56 \%$ (5) presentó CaP Gleason $6(3+3)$ y el $44 \%$ (4) Gleason 7 (3+4). El 59\% (20) se incluyeron en el Grupo

\section{CORRESPONDENCIA}

Jeff R. Cortés-González

Servicio de Urología. Hospital Universitario Dr. José E. Gonzalez. UANL.

Monterrey. Nuevo León. (Méjico).

jrcor77@yahoo.com

jeff.cortes-gonzalez@ki.se

Aceptado para publicar: 22 de octubre 2009
1 y $41 \%$ en el Grupo 2. En el grupo 1 16\%(3) presentaron CaP vs 46\% (6) del grupo 2 (p 0.04), RR=3.07.

CONCLUSIÓN: Hay indicios de que la tasa de detección del CaP en la población de estudio pueda ser menor a lo reportado en la literatura. El TR es importante en la evaluación inicial, pequeñas anormalidades incrementan 3 veces el riesgo de CaP.

Palabras clave: Cáncer de próstata. Antígeno prostático específico. Biopsia transrectal.

Summary.- OBJECTIVES: To find the detection rate of prostate cancer (PCa) in our population with PSA values between 2.6 and $4 \mathrm{ng} / \mathrm{ml}$.

METHODS: We included 33 consecutive patients with a median age of 66 years, that had a Transrectal Ultrasound (TRUS) guided biopsy with PSA between 2.6-4 $\mathrm{ng} / \mathrm{ml}$. Patients were divided into 2 groups. Group 1: patients with normal Digital Rectal Examination (DRE) and Group 2: Patients with DRE with asymmetry not definitive of PCa. Exclusion criteria: known history of PCa, intraepithelial neoplasia or Positive DRE. Statistical analysis: Chi square, t-student and Fischer exact test.

RESULTS: Twenty eight percent of the patients (9) had positive biopsy for PCa. Fifty six percent (5) were Gleason 6 and 44\% (4) Gleason 7 (3+4). Group 1 had 59\% (20) and Group $241 \%$ in. In Group 1 16\% (3) had positive biopsy for PCa vs 46\% (6) in group 2 (p 0.04) RR 3.07.

CONCLUSIONS: There are traces that the detection rate in our population could be lower in comparison with what has been reported in the literature. DRE is crucial in the initial evaluation; asymmetry could increase 3 fold the risk of having PCa. 
Keywords: Prostatic cancer. PSA. Biopsy.

\section{INTRODUCCIÓN}

El límite superior de normalidad en el Antígeno Prostático Especifico (APE) que es comúnmente usado en la práctica clínica es el de $4.0 \mathrm{ng} / \mathrm{ml}$. $(1,2)$. Este punto de corte es incapaz de identificar alrededor de $25-30 \%$ de pacientes con cáncer de próstata (CaP) con APE entre 2.6 y $4.0 \mathrm{ng} / \mathrm{ml} .(2,3)$. Se estima que alrededor de $80 \%$ de los especímenes de estos pacientes posterior a la prostatectomía radical tengan enfermedad confinada a la glándula. La presentación del CaP es muy variada en el mundo (4), siendo más alta en los países escandinavos y más baja en los países orientales como China. La incidencia en países emergentes como lo son algunos países de Latinoamérica es incierta debido a la falta de regulación en su registro $(4,5)$. Debido a esta falta de registro en países latinoamericanos, decidimos observar la tase de deteccion de cáncer de próstata en la población que acude a nuestro hospital con APE entre los rangos de 2.6 y $4.0 \mathrm{ng} / \mathrm{ml}$ y compararla con lo reportado en la literatura mundial.

\section{MATERIAL Y MÉTODOS}

De julio del 2006 a julio del 2008 se incluyeron 33 pacientes consecutivos entre 50 y 79 años (mediana de 66) que acudieron a nuestro servicio para valoración de hiperplasia prostática benigna, que dentro de su valoración presentaban un APE entre 2.6 y $4.0 \mathrm{ng} / \mathrm{ml}$ y que tras una explicación breve aceptaron participar en nuestro estudio. El estudio fue debidamente registrado y aceptado por parte del comité de ética del hospital Universitario "Dr. José E. González" de la Universidad Autónoma de Nuevo León y a todos los pacientes se les entregó una forma de consentimiento informado para su inclusión. Como parte de la evaluación, todos los pacientes fueron sometidos a examen físico prostático mediante tacto rectal (TR). Esta evaluación fue hecha por 3 urólogos adscritos a nuestra institución. Las personas con algún valor previo de APE $>4 \mathrm{ng} / \mathrm{ml}$, diagnostico de CaP, neoplasia intraepitelial, antecedentes de resección transuretral de próstata o datos clínicos al TR evidentes de CaP fueron excluidos de este estudio. Una vez que el paciente aceptó participar, se le realizó una biopsia transrectal guiada por ultrasonido (BTR-US), lo cual se efectuó con anestesia local (jalea lubricante + xilocaína intrarectal por $5 \mathrm{~min}$ ). El esquema sobre el número de cilindros, que rutinariamente se realiza en nuestro servicio, es de $8(n=11)$ ó $10(n=16)$, aunque en este análisis se incluyeron 4 pacientes con 6 y dos con 12 (mediana 9). Todos los cilindros fueron enviados al servicio de anatomía patológica de nuestro hospital y fueron evaluados por un patólogo. Los pacientes se dividieron en 2 grupos. El grupo 1: pacientes con TR normal y el grupo 2: pacientes con ligero aumento en la consistencia de la próstata mas no evidencia franca de neoplasia. La mediana de edad fue de 65 y 66 años en ambos grupos, respectivamente (valor de p 0.34). La prueba t de student se realizó para evaluar diferencias en las medias. La prueba $X^{2}$ y la de prueba exacta de Fisher para evaluar diferencias en las frecuencias. Valores de la $p \leq 0.05$ fueron considerados estadísticamente significativos.

\section{RESULTADOS}

No se observó ninguna complicación grave posterior a la BTR-US. El $28.3 \%$ (9) del total de pacientes en este estudio presentó biopsia positiva para el diagnostico histopatológico de CaP. El 56\% (5) de estos pacientes presentó Gleason $6(3+3)$, el $44 \%$ (4) Gleason $7(3+4)$ y no se observaron pacientes con Gleason $\geq 8$. El $59 \%$ (20) tuvieron TR normal y fueron incluidos en el Grupo 1 por lo que el $41 \%$ (13) restante presentaron ligero aumento en la consistencia de la próstata sin evidencia franca de neoplasia y se incluyeron en el Grupo 2. En el Grupo 1, 16\% (3) presentaron diagnóstico histopatológico de $\mathrm{CaP}$ como se observa en la Tabla I. En comparación con el $46 \%$ (6) del grupo 2. Esta diferencia fue estadísticamente significativa (valor de la $\mathrm{p} 0.04$ ), con un riesgo relativo de 3.07 (IC 95\% 1.00-9.90) como se observa en la Figura 1. No hubo diferencias significativas en la detección de casos de CaP en pacientes mayores y menores de 70 años (valor de p 0.69) en la población total del estudio. Lo mismo fue observado en el grupo 1 (valor de la p 0.14), sin embargo, en el grupo 2, la detección en los pacientes $>70$ fue menos frecuente (valor de la p 0.04).

\section{DISCUSIÓN}

El APE continua utilizándose de manera rutinaria en la práctica urológica en todo el mundo (2). Aun así, existe el debate sobre el valor de corte para indicar la BTR-US (6). Thompson et al reportaron que aproximadamente un $29 \%$, independientemente de la edad o raza, tenían CaP cuando el APE se encontraba entre 3.1 y $4.0 \mathrm{ng} / \mathrm{ml}$ (7) y Pepe et al. (8) el $26.4 \%$ con APE entre 2.6 y $4 \mathrm{ng} / \mathrm{ml}$, ambos con TR normal y biopsias por sextantes. En nuestro estudio encontramos que el $28.3 \%$ presentó CaP, sin embargo, $39.4 \%$ de los pacientes tenían TR ligeramente anormal, así como el esquema de BTR-US fue con mayor numero de biopsias. Nadler et al. (6) reportaron 


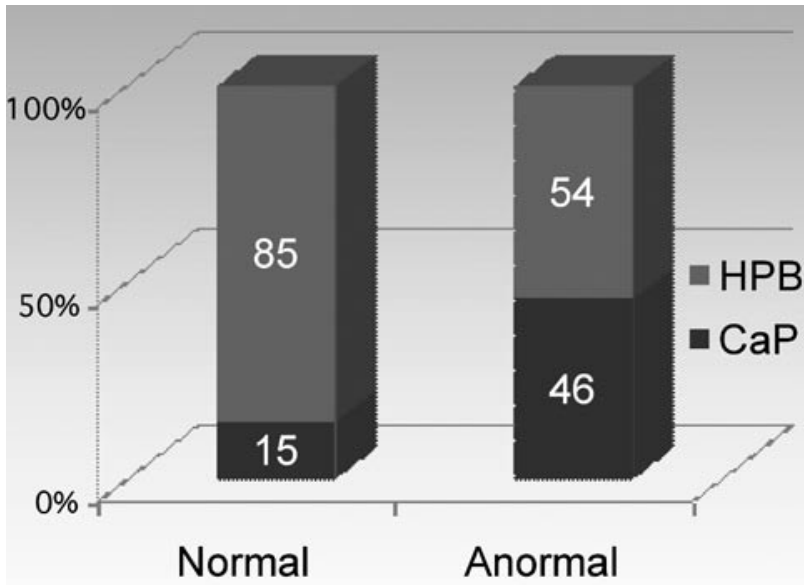

FIGURA 1. Incidencia de CaP acorde al Tacto Rectal.

$16 \%$ de CaP con APE entre 2.6 y $4 \mathrm{ng} / \mathrm{ml}$ con BTR-US de sextantes en pacientes mayores de 60 años con TR normal y un pequeño porcentaje de pacientes con TR anormal. En nuestro estudio, el CaP fue detectado en el 16\%, cuando el TR era normal. En ese mismo estudio, Nadler et al. (6) encontraron una tendencia en la edad $>70$ con un valor predictivo positivo bajo para la detección de CaP con APE en estos rangos, interesantemente no se presentó detección de $\mathrm{CaP}$ en pacientes $>70$ en aquellos con TR ligeramente anormal (grupo donde el riesgo fue 3 veces mayor). Con estos resultados, pudiéramos especular que la tasa de detección de CaP podría ser menor en nuestra población en pacientes menores de 70 años y TR normal ya que en nuestro estudio el porcentaje de pacientes con CaP pudiera estar acrecentado por el gran número de pacientes con TR ligeramente anormal, además, el esquema de biopsias fue más agresivo que los otros estudios y esto pudiera también incrementar el número de detecciones de CaP (9). Sin embargo, esto solo podría ser respondido en un estudio multicentrico con una mayor muestra de pacientes.

Aún a pesar de que algunos centros han adoptado y recomiendan la medida de tomar biopsias cuando el APE es $>2.5 \mathrm{ng} / \mathrm{ml}(6,10)$, el punto de corte aun no está determinado (11) y algunos autores recomiendan la BTR-US de manera individualizada cuando el valor se encuentra por arriba de $2.5 \mathrm{ng} /$ $\mathrm{ml}$ y el TR es normal, ya que es un procedimiento invasivo y en ocasiones innecesario $(12,13)$. El futuro debería centrarse en la investigación de marcadores mas fidedignos que nos ayuden a reducir el número de biopsias innecesarias. Ya que el hecho de incrementar el número de cilindros obtenidos en cada BTR-US y disminuir el punto de corte del APE, podría llevar a un aumento en el número de detecciones, llevando a un sobre diagnostico y sobre tratamiento de $\mathrm{CaP}$ de bajo riesgo y/o insignificante, es decir casos de CaP que no ocasionarían ningún riesgo ulterior al paciente a lo largo de su vida (14).

Una de las desventajas de nuestro estudio es la muestra tan pequeña de pacientes y un posible sesgo regional, ya que fue realizado solo en la región noreste de México, por lo cual no es posible saber la real incidencia de CaP en todo el país en el

TABLA I. CARACTERISTICAS DE LOS PACIENTES CON DIAGNOSTICO DE CAP.

\begin{tabular}{|c|c|c|c|c|c|}
\hline Paciente & Edad & APE & TR & Gleason & No. cilindros \\
\hline I & 64 & 3.5 & anormal & 7 & 10 \\
\hline$I I$ & 64 & 3.36 & anormal & 6 & 10 \\
\hline III & 66 & 3.3 & anormal & 6 & 8 \\
\hline IV & 64 & 3.49 & anormal & 6 & 10 \\
\hline$V$ & 57 & 2.8 & anormal & 7 & 10 \\
\hline$V I$ & 66 & 2.6 & anormal & 7 & 8 \\
\hline VII & 79 & 3.7 & normal & 6 & 10 \\
\hline VIII & 76 & 3.2 & normal & 6 & 10 \\
\hline IX & 63 & 4 & normal & 7 & 6 \\
\hline
\end{tabular}


rango estudiado del APE y mucho menos extrapolarlo al resto de los países latinoamericanos. Además, de que el número de cilindros por BTR-US no fue homogéneo. Sin embargo, pudiera motivar la realización un estudio multicéntrico que incluyan personas con TR normal entre un rango de edad 50 y los 70 años para validar externamente las recomendaciones adoptadas por algunos centros de excelencia así como las guías europeas y americanas para poder extrapolarlas a nuestra población.

Históricamente existen diversos tabús en algunas culturas latinoamericanas en las que los pacientes no permiten al explorador realizar el TR por temor a perder su masculinidad (15) por lo que, en nuestra experiencia, sugieren al médico tratante solicitarles solo el examen de sangre (APE) y ya una vez que el resultado sea "anormal" entonces podrían aceptar el examen rectal. Para refutar estar practica en nuestro medio, en un esfuerzo por producir medicina basada en evidencias, se puede observar que el hecho de que a la exploración física se encuentre cualquier anormalidad por mínima que esta sea, el riesgo es 3 veces mayor de tener $\mathrm{CaP}$ cuando el antígeno se encuentra en estos rangos. Hasta el momento no existe en nuestro conocimiento ningún estudio realizado en algún país de Latinoamérica que evalué este rubro.

\section{CONCLUSIONES}

No se puede llegar a conclusiones contundentes en este estudio con una muestra tan pequeña. Sin embargo hay indicios de que la tasa de detección del CaP en personas con TR normal y APE entre 2.6 y $4 \mathrm{ng} / \mathrm{l}$ en la población de estudio pueda ser menor a lo reportado en la literatura. El TR es indispensable en la evaluación inicial del paciente, ya que incrementa 3 veces el riesgo de CaP si se asocia con asimetría o ligero aumento en la consistencia de la próstata aún en rangos de APE que en general se consideran normales en algunos centros. Se requiere de un estudio prospectivo, multicéntrico en personas con TR normal entre edades de 50-70 años y con BTR-US homogéneo para validar externamente las recomendaciones de algunos centros de excelencia, de las guías europeas y americanas para poder extrapolarlas a nuestra población.

\section{BIBLIOGRAFÍA y LECTURAS RECOMENDADAS (*lectura de interés $y^{* *}$ lectura fundamental)}

1. Roehl K A, Antenor J A V and Catalona W J. Serial biopsy results in prostate cancer screening study. $\mathrm{J}$ Urol, 2002; 167: 2435.
**2. Eggener SE, Roehl K, Catalona WJ. Predictors of subsequent prostate cancer in men with a prostate specific antigen of 2.6 to $4.0 \mathrm{ng} / \mathrm{ml}$ and an initially negative biopsy J Urol, 2005; 174, 500-4.

3. Zackrisson B, Aus G, Lilja H, Lodding P, Pihl C G and Hugosson J. Follow-up of men with elevated prostate-specific antigen and one set of benign biopsies at prostate cancer screening. Eur Urol, 2003; 43: 327.

4. Zeigler-Johnson CM, Rennert H, Mittal RD, Jalloh M, Sachdeva R, Malkowicz SB, Mandhani A et al. Evaluation of prostate cancer characteristics in four populations worldwide. Can J Urol, 2008; 15(3):4056-64.

5. Haas GP, Delongchamps N, Brawley OW, Wang CY, de la Roza G. The worldwide epidemiology of prostate cancer: perspectives from autopsy studies. Can J Urol, 2008; 15(1):3866-71.

*6. Nadler RB, Loeb S, Roehl KA, Antenor JA, Eggener $\mathrm{S}$, CatalonaWJ Use Of $2.6 \mathrm{ng} / \mathrm{ml}$ Prostate Specific Antigen Prompt For Biopsy In Men Older Than 60 Years J Urol Vol, 2005; 174, 2154-7.

7. Thompson I M, Pauler D K, Goodman P J, Tangen C M, Lucia M S, Parnes H L et al. Prevalence of prostate cancer among men with a prostate-specific antigen level or $4.0 \mathrm{ng}$ per milliliter. N Engl J Med, 2004; 350: 2239.

8. Pepe P, Panella P, Savoca F, Cacciola A, D'Arrigo L, Dibenedetto G, Pennisi M, Aragona F. Prevalence and clinical significance of prostate cancer among 12,682 men with normal digital rectal examination, low PSA levels $(<$ or $=4 \mathrm{ng} / \mathrm{ml}$ ) and percent free PSA cutoff values of 15 and 20\%. Urol Int, 2007; 78(4):308-12.

9. Gore JL, Shariat SF, Miles BJ, Kadmon D, Jiang N, Wheeler TM, Slawin KM. Optimal combinations of systematic sextant and laterally directed biopsies for the detection of prostate cancer. J Urol. 2001; 165(5):1554-9.

10. Catalona WJ, Loeb S, Han M. Viewpoint: expanding prostate cancer screening. Ann Intern Med. 2006; 144(6):441-3.

*11. Aus G, Abbou CC, Bolla M, et al. EAU guidelines on prostate cancer. Eur Urol, 2005; 48:546-551.

*12. Pepe P, Panella P, D’Arrigo L, Savoca F, Pennisi M, Aragona F Should Men with Serum Prostate-Specific Antigen $4 \mathrm{ng} / \mathrm{ml}$ and Normal Digital Rectal Examination Undergo a Prostate Biopsy? Oncol, 2006; 70(2):81-9.

13. Lucia MS, Darke AK, Goodman PJ, La Rosa FG, Parnes HL, Ford LG, Coltman CA Jr, Thompson IM. Pathologic characteristics of cancers detected in The Prostate Cancer Prevention Trial: implications for prostate cancer detection and chemoprevention. Cancer Prev Res (Phila Pa). 2008; 1(3):167-73.

14. O'Donnell H, Parker C. What is low-risk prostate cancer and what is its natural history? World J Urol. 2008 Oct;26(5):415-22. Epub 2008 Jun 21. Review.

15. Cannen A. Peña Melo, Evelyn P. Ulloa O., Grisel Garcla Felipe, Yudania Vásquez, Luis Quezada Actitudes respecto al tacto rectal en pacientes masculinos que acuden a la consulta externa del Hospital Juan Pablo Pina, en el período Abril-Junio 2000 Rev. Med. Dom. DR-ISSN-0254-4504 ADOERBIO 001 Vol.64 (3) y Vol.65(1) 2004 